\title{
Curie Temperature of Low-dimensional Ferromagnetic Material
}

\author{
Shuai Zhang \\ College of Materials Science and Engineering, Jilin JianZhu University, Changchun 130118, China
}

Corresponding Author Email: zhangshuaixx@ sina.com

https://doi.org/10.18280/ijht.370111

Received: 19 September 2018

Accepted: 11 December 2018

\section{Keywords:}

low dimension, crystal, curie temperature

\begin{abstract}
This paper attempts to develop a unified theoretical model to describe the size dependence of the Curie temperature for materials of different sizes. To this end, the size-dependence model of glass transition temperature was expanded into a size-dependence model of the Curie temperature Tc of uniform low-dimensional ferromagnetic materials, based on energy theory and thermodynamics. The proposed model focuses on the critical size and the material shape, and has no adjustable free parameter. The model was used to predict the effect of material size on the Curie temperature, revealing that the Tc value decreases with the material size. This result agrees well with the experimental results and the prediction by other theoretical models. The research findings make up for the gap in the previous research on the Curie temperature.
\end{abstract}

\section{INTRODUCTION}

With reduced symmetry, ferromagnetic nanometals have different properties from bulk metals. The size of these materials has direct impacts on local surface properties like valence band structure and membrane shape. In return, these properties can serve as sensitive functions of material size and growth conditions [1].

In terms of application, a nanocrystal can be divided into two categories, namely, a thin membrane less than $10 \mathrm{~nm}$ in thickness [2-3] and a bulk material consisting of nanoparticles or nanocrystals [4]. In recent years, much research has been done on the magnetic properties of membranes, especially the temperature of magnetic phase transition, i.e. the Curie temperature [2-3], revealing that the Curie temperature changes with the material size. On this basis, some theoretical models have been developed to describe the variation of Curie temperature with sizes [1-3].

One of these models is grounded on magnetic pole coupling. For a thick membrane, the Curie temperature satisfies:

$$
T_{\mathrm{c}} / T_{\mathrm{c} 0} \approx 1-\left[\left(n_{0}+1\right) / 2 n\right]^{\lambda}
$$

where Tc0 is the Curie temperature of the bulk crystal; $\lambda \in[1$, 1.59] [2] is a constant dependent on the magnetic pole coupling; $\mathrm{n}=\mathrm{S} / \mathrm{h}$ is the number of deposited membrane layers (MLs), with $\mathrm{S}$ being the membrane thickness and $\mathrm{h}$ being the layer spacing (i.e. atomic diameter). If the membrane thickness becomes sufficiently small n0, there will be a linear relationship between the Curie temperature and the material size:

$\frac{T_{C}}{T_{c 0}}=\frac{n-1}{2 n_{0}}$

The above model predicts that Curie temperature decreases with the membrane thickness, which has been validated by experimental results.
Another model, created on the sequence, length and strength of keys [5], can also accurately depict the Curie transition of ferromagnetic membranes.

Meanwhile, the unique physical-chemical properties of ferromagnetic particles have also attracted attention in basic and applied research. For these particles, the Curie transition is essentially the shift of magnetic domains from disordered alignment to ordered alignment. The shift takes place right after the decline in particle size $D$, a.k.a. particle/grain diameter. To our knowledge, there is no theoretical model on the size dependence of the Curie temperature of ferromagnetic particles, which differs from that of membranes [2]. Thus, it is necessary to develop a unified theoretical model for particles with various sizes $d$.

The previous studies have shown the dependence of Curie transition on the material shape and size [2]. As a result, the physical nature of the transition should be fully considered in both theory and application.

In addition, the Curie transition is a secondary phase transition. Its features can be derived from those of other secondary phase transitions like glass transition. For glass transition, the size-dependence of the transition temperature $T_{\mathrm{g}}$ has been obtained for various low-dimensional materials by extending the size-dependence model of the melting temperature [6-7].

In this paper, the size-dependence model of glass transition temperature is expanded to examine the size dependence of the Curie temperature for magnetic materials in different sizes. Special attention was paid to determining the critical size $D_{0}$, i.e. the size of a single-domain particle/domain. The determination requires different methods for different material sizes. Several experiments were carried out on $\mathrm{Fe}, \mathrm{Co}$ and $\mathrm{Ni}$ membranes as well as Ni nanoparticles and Ni nanowires. The prediction of our model was found to be consistent with the experimental results. 


\section{MODELLING}

Replacing the glass transition temperature $T_{\mathrm{g}}$ and bulk material transition temperature $T_{\mathrm{g} 0}$ with the Curie temperature $T_{\mathrm{c}}$ and bulk material Curie temperature $T_{\mathrm{c} 0}$ [6-7], the glass transition temperature $T_{\mathrm{g}}$ model can be modified as:

$\frac{T_{c}}{T_{c 0}}=\exp \left(-\frac{2 \Delta C_{p}}{3 R} \frac{1}{\left(\frac{D}{\left.D_{0}-1\right)}\right.}\right)$

where, $\Delta C_{p}$ is the specific heat difference of the ferromagnetic body at the Curie temperature $T_{c 0} ; R$ is the ideal gas constant; $D_{0}$ is the critical size of the material that induces the Curie transition.

The critical size $\mathrm{D}_{0}$ is usually defined as the diameter of all atoms on the surface of a low-dimensional material. If the dimension $\mathrm{k}$ of a spherical particle is zero, then the critical size $\mathrm{D}_{0}$ equals six times the atomic diameter $\mathrm{h}$; if $\mathrm{k}=1$ (i.e. the material is a nanowire), then the critical size equals four times the atomic diameter; if $\mathrm{k}=2$ (i.e. the material is a membrane), then the critical size equals twice the atomic diameter.

The material size $D$ directly bears on the magnetic transition of low-dimensional magnetic materials. The magnetic transition takes place when the temperature reaches a critical value $T_{\mathrm{c}}$, turning the ferromagnetic body into paramagnetic body. In other words, the magnetic domains change from ordered alignment to disordered alignment. The magnetic transition is reversible. If the temperature decreases, the paramagnetic body will change back into ferromagnetic body, and the magnetic domains will become more orderly. Here, $2 D_{0}$ is defined as the minimum material size for magnetic domains to remain orderly.

With low magnetic permeability and high coercive force, single-domain particles have a great influence on material properties and critical size estimation. These particles can only be magnetized rotationally, due to the absence of domain walls, and cannot be easily magnetized or demagnetized without external field or external force.

The critical size is the division point between the singledomain and other domain structures. Thus, the energy of the critical size of the single-domain structure is comparable to that of the simplest adjacent multi-domain structure. The two structures will have equal energy if both are at the critical size. Thus, the critical size of a single domain can be identified using the comparability [8].

The domains in ultrafine particles are spherical. For such a spherical domain, the total energy can be calculated as $E_{\mathrm{T}}=E_{\mathrm{w}}+E_{\mathrm{H}}+E_{\mathrm{d}}$, where $E_{\omega}$ is the domain wall energy, $E_{\mathrm{H}}$ is the magnetostatic energy, and $E_{\mathrm{H}}$ is the demagnetizing energy $E_{\mathrm{d}}$ [9]. Without external magnetic field, there is naturally no magnetostatic energy. The demagnetizing energy can be neglected because the domain surface only has a weak magnetic pole. Hence, the total energy of a spherical domain without external magnetic field is equivalent to the domain wall energy [9]:

$E_{\mathrm{T}}=E_{\omega}=S \sigma / 2$

where, $S=\pi D^{2}$ and $\sigma$ are the area and the energy density of the domain wall, respectively; $D$ is the diameter of the domain. The energy density varies from domain wall to domain wall.

For the $90^{\circ}$ domain wall in cubic crystals, $\sigma$ equals $\pi \sqrt{A_{1} K_{1}} / 2$; for the $180^{\circ}$ domain wall in uniaxial crystals, $\sigma$ equals $4 \sqrt{A_{1} K_{1}}$ [10]. Here, $A_{1}$ is the exchange integral constant and $K_{1}$ is the magnetic anisotropy constant.

For single-domain crystals, the magnetocrystalline anisotropy energy is minimized when the magnetic moments are arranged in parallel along the easy magnetization axis. In the absence of external magnetic field and internal stress, neither the magnetostatic energy nor magnetoelastic energy of the external field needs to be considered. Coupled with the lack of energy exchange, it is only necessary to consider the demagnetizing energy. Hence, the total energy of a singledomain crystal can be expressed as [10]:

$E_{\mathrm{T}}=E_{\mathrm{d}}=\mu_{0} V N M_{\mathrm{s}}^{2} / 2$

where, $\mu_{0}$ is the magnetic constant or a vacuum permeability; $M_{\mathrm{s}}$ is the saturation magnetization intensity; $V=\pi D^{3} / 6$ is the volume of the domain; $N$ is the demagnetization factor. Here, $N=N_{\mathrm{a}} \alpha_{\mathrm{a}}^{2}+N_{\mathrm{b}} \alpha_{\mathrm{b}}^{2} N_{\mathrm{c}} \alpha_{\mathrm{c}}^{2}$, with $\alpha_{\mathrm{i}}$ (i=a, b, c, the three major axes of the crystal) being the direction cosine of the saturation magnetization intensity $M_{\mathrm{s}}$, and $N_{\mathrm{a}}, N_{\mathrm{b}}$ and $N_{\mathrm{c}}$ being the demagnetization factors along the three axes $\left(N_{\mathrm{a}}+N_{\mathrm{b}}+N_{\mathrm{c}}=1\right)$, respectively. For spherical particles, the major axes are equal in length $(\mathrm{a}=\mathrm{b} \quad \mathrm{c})$ such that $N_{\mathrm{a}}=N_{\mathrm{b}}=N_{\mathrm{c}}$. Thus, the demagnetization factor $N$ of a spherical particle equals $1 / 3$ [10]. According to formulas (2) and (3), the critical size $D_{0}$ can be derived as:

$D_{0}=\frac{9 \sigma}{\mu_{0} M_{S}^{2}}$

The size dependence of the Curie temperature for magnetic nanoparticles can be obtained from formulas (3) and (6). It can be seen from the formulas that the Curie temperature increases exponentially with the reduction in particle size.

For wires and membranes, the ferromagnetic coupling is sufficiently strong to make the magnetic momentum parallel to the membrane direction [1], when the wire/membrane thickness $n$ (the number of atomic layers) is greater than 20 30. Therefore, the size dependence of the Curie temperature $T_{\mathrm{c}}$ for the wire/membrane can be predicted directly by formulas (3) and (6).

If $n$ is smaller than 20 30, most ferromagnetic membranes will change from 2-dimension to quasi-1-dimension [11]. In this case, the membranes exist as an island-like deposition on the substrate, and the critical size $D_{0}$ should be recalculated. For these membranes, the domain size is no longer the membrane thickness, but the diameter of island-like membrane.

By first-order approximation, the shape of island-like membrane can be viewed as a flat sphere or a spherical crown. Then, we have $S=\pi D^{\prime} t, V=\pi\left(D^{\prime}\right)^{2} t / 4$, with $D^{\prime}$ and $t$ being the diameter and thickness of the flat spherical domain, respectively. As discussed above, $D_{0}^{\prime}$ equals $2 \sigma /\left(\mu_{0} N M_{\mathrm{s}}{ }^{2}\right)$ if $E_{\omega}=E_{\mathrm{d}}$. The island-like membrane can be considered as a quasi-1D system, because it is an intermediate form between particle (0D) and thin membrane (2D) [94]. Then, the demagnetization factor $N$ of the island-like membrane equals the arithmetic mean (2/3) of the demagnetization factor $(\mathrm{N}=1 / 3)$ of the particle and the demagnetization factor $(N=1)$ of the membrane.

The relationship between $D^{\prime}$ and $D$ can be determined according to the Gibbs free energy difference $\Delta \mathrm{G}$ between the island-like membrane and the normal membrane [94]. 
Here, a square membrane (side length: $L$; thickness: $D$; volume: $V=L^{2} D$ ) is cited as an example for the calculation of $\Delta G$. Under a certain temperature $T$, the membrane will transform into a flat spherical particle of the same volume. In this case, the Gibbs free energy difference $\Delta G$ can be calculated as $V \Delta P_{0}+\left(\gamma_{2} S_{2}-\gamma_{1} S_{1}\right)$, where, $\Delta P_{0}=P_{1}-P_{0}$ is the pressure difference between the island-like membrane and the normal membrane; $S_{2}=\pi\left(D^{\prime}\right)^{2} / 4+\pi\left(D^{\prime}\right)^{2} / 4+\pi D^{\prime} t$ is the sum of the surface area of the island-like membrane and the area of the interface between the membrane surface and the substrate; $S_{1}=L^{2}+L^{2}+4 L D$ is the sum of the surface area and the interface area before the formation of the island-like membrane; $\gamma_{1}$ and $\gamma_{2}$ are the surface energies (interface energies) before and after the transition, respectively. Note that $P_{1}$ refers to the pressure difference between the inside and outside of the membrane resulted from the surface curvature of the island-like membrane. According to the Gibbs-Thomson equation, $P_{1}$ equals $2 f / D^{\prime}$, with $f$ being the surface stress of the crystal [12]. For the normal membrane, $P_{0}$ equals zero as the surface is flat with no curvature. In addition, the first and second terms in $S_{2}$ represent the surface area of the upper portion and the interface area between the lower portion and the substrate, respectively.

Thus, it can be derived that $\Delta G=V\left(2 f / D^{\prime}+\gamma / t+\gamma^{\prime} / t+4 \gamma / D^{\prime}-\gamma / D\right.$ $\left.\gamma^{\prime} / D-4 \gamma / L\right)$, where $\gamma$ is the surface energy of the crystal; $\gamma^{\prime}$ is the solid interface energy between the membrane and the substrate. Considering the material difference across the interface between the membrane and the substrate, the value of $\gamma^{\prime}$ can be computed by calculating the solid interface energy between the same materials and taking the arithmetic mean as the solid interface energy between two different materials. The values of $f$ and $\gamma_{\mathrm{ss}}$ can be calculated as [13]:

$f= \pm \frac{7}{2\left(\frac{T_{m}}{T+6)}\right.} \sqrt{3 h^{2} S_{v i b} H_{m} /\left(\kappa R V_{m}\right)}$

$\gamma_{s S}=\frac{196 h T^{2} H_{m} S_{v i b}}{3 R\left(T_{m}+6 T\right)^{2} V_{m}}$

where $T_{\mathrm{m}}$ is the melting point of the material; $h$ is the atomic diameter; $H_{\mathrm{m}}$ is the melting enthalpy; $S_{\text {vib }}$ is the vibrational entropy in the melting entropy $S_{\mathrm{m}}\left(S_{\mathrm{vib}} \approx S_{\mathrm{m}}\right.$ for metals [63]); $R$ is the ideal gas constant; $V_{\mathrm{m}}$ is the molar volume; $\kappa=1 / B$ is the compression factor, with $B$ being the bulk modulus.

Once the membrane thickness is reduced to a certain extent, the uniform membrane will spontaneously transform into the island-like membrane if the Gibbs free energy difference $\Delta G_{0}$ between the two membranes is below zero. Mathematically, the spontaneous transition will take place if $\Delta G \leq 0$. Since $V=L^{2} D=\pi\left(D^{\prime}\right)^{2} t / 4$ and $L=D^{\prime}$ at the critical point, it is possible to deduce that $D=\pi t / 4$. Then, the transition condition can be written as $D^{\prime} / D \geq 8 f /\left[(4-\pi)\left(\gamma+\gamma^{\prime}\right)\right]$. Assuming that $D^{\prime} / D=C$, we have:

$C=8 f /\left[(4-\pi) /\left(\gamma+\gamma^{\prime}\right)\right]$

Substituting $D^{\prime} / D=C$ into $D_{0}^{\prime}=\sigma /\left(\mu_{0} N M_{\mathrm{s}}^{2}\right)$, we have:

$D_{0}=\frac{3 \sigma}{\mu_{0} C M_{S}^{2}}$

\section{RESULTS AND DISCUSSION}

The $T_{c}$ curve of Ni particles and wires is illustrated in Figure
1, where the solid line depicts the $T_{\mathrm{c}}$ curve of Ni particles and wires obtained by formulas (3) and (6), the solid dot stands for the experimental results of Ni particles [4], and the hollow dots and plus sign represent the experimental results of $\mathrm{Ni}$ wires $[14,15]$.

Considering the $90^{\circ}$ domain walls of Ni particles and wires, the value of $\sigma$ must be $\pi \sqrt{A_{1} K_{1}} / 2$. For large nanowires, the size dependence of the Curie temperature can also be theoretically obtained from formula (3), after redefining $D$ as the diameter of the nanowire [14]. As shown in Figure 1, both the theoretical and experimental results agree that $T_{c}$ decreases with the particle size $D$.

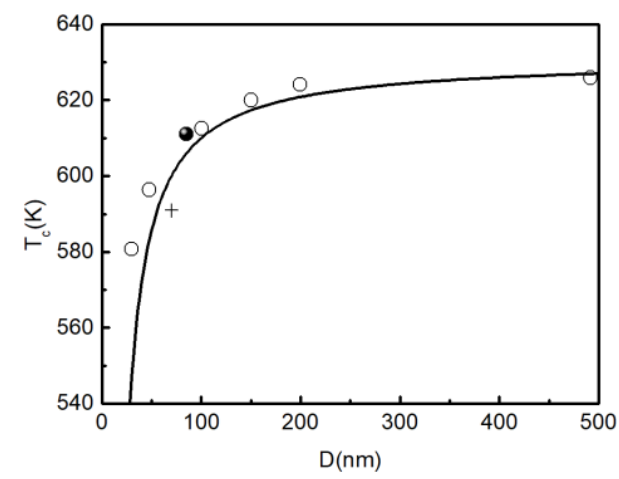

Figure 1. The $T_{c}$ curve of Ni particles and wires

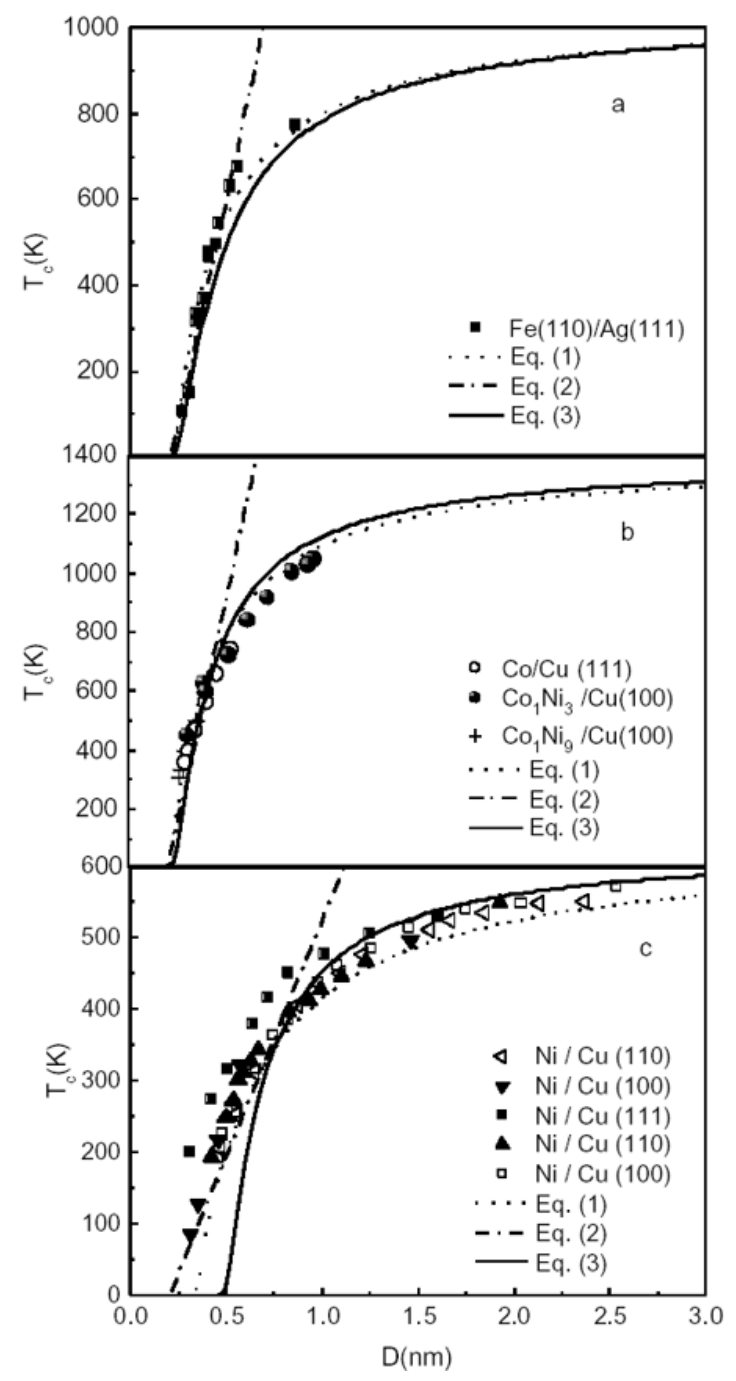

Figure 2. The $T_{\mathrm{c}}$ curves of $\mathrm{Fe}, \mathrm{Co}$ and $\mathrm{Ni}$ membranes 
The $T_{c}$ curves of $\mathrm{Fe}, \mathrm{Co}$ and $\mathrm{Ni}$ membranes deposited on different substrates with coherent interfaces are shown in Figure 2, where the solid lines stand for the results predicted by formulas (3) and (10); the dash lines reflect the results predicted by formulas (1) and (2); the solid boxes represent the experimental results for Fe [23]; the solid dots, hollow dots and plus sign jointly describe the experimental results for Co [24]; the solid up arrows, hollow boxes, solid left arrows, solid down arrows and solid boxes joints represent the experimental results for Ni [25-27].

Considering the $180^{\circ}$ domain walls of the membranes, the value of $\sigma$ must be $4 \sqrt{A_{1} K_{1}}$. As shown in Figure 2, the theoretical predictions on the $T_{c}$ of $\mathrm{Fe}, \mathrm{Co}$ and Ni membranes agree well with the experimental results, indicating that the material size changed from 2-dimension to quasi-1-dimension with the reduction in membrane thickness. Since the magnetic order relies on material size, it is concluded that the dimensional transition has a certain impact on the ordering of magnetic domains. In addition, the $T_{\mathrm{c}}$ of membranes declined much faster than that of large particles and wires. This is because the island-like membrane has a much smaller $D_{0}$ than particles.

Compared with the model based on spin interaction (formulas (1) and (2)), formula (3) can predict the theoretical value of $T_{\mathrm{c}}$ excellently using the unified $T_{\mathrm{c}}$ equation only. Moreover, that formula (3) has no free parameter, while both $n_{0}$ and $\lambda$ are fitting parameters in formulas (1) and (2).

Our model also discloses the relationship between membrane shape and membrane thickness from the angle of thermodynamics. The low surface-to-volume ratio was found to be the primary cause for the membrane to change from a uniform 2-dimension material to an island-like membrane. The model can also reveal the size dependence of ferroelectric transitions, after redefining $2 D_{0}$ as the transition limit [28].

Table 1. Parameters and data in Figures 1 and 2

\begin{tabular}{cccc}
\hline & $\mathrm{Fe}$ & $\mathrm{Co}$ & $\mathrm{Ni}$ \\
\hline$T_{\mathrm{c} 0}(\mathrm{~K})[18]$ & 1042 & 1395 & 631 \\
$A_{1}\left(10^{-11} \mathrm{~J} / \mathrm{m}\right)[18]$ & 1.21 & 1.5 & 0.67 \\
$K_{1}\left(\mathrm{~J} / \mathrm{m}^{3}\right)[18]$ & $4.8 \times 10^{4}$ & $4.3 \times 10^{4}$ & $-4.5 \times 10^{3}$ \\
$M_{\mathrm{s}}\left(\mathrm{A} / \mathrm{m}^{-1}[16]\right.$ & $1.712 \times 10^{6}$ & $1.422 \times 10^{6}$ & $4.85 \times 10^{5}$ \\
$\Delta C_{\mathrm{p}}\left(\mathrm{Jg}-\mathrm{atom} \mathrm{K}^{-1}\right)[19]$ & 15.24 & 10.9 & 5.06 \\
$h(\mathrm{~nm})[20]$ & 0.344 & 0.334 & 0.324 \\
$V_{\mathrm{m}}\left(\mathrm{cm}^{3} \mathrm{~mol}^{-1}\right)[20]$ & 7.1 & 6.7 & 6.59 \\
$H_{\mathrm{m}}\left(\mathrm{KJ} \mathrm{mol}^{-1}\right)[20]$ & 13.80 & 16.19 & 17.47 \\
$T_{\mathrm{m}}(\mathrm{K})[20]$ & 1809 & 1768 & 1726 \\
$S_{\mathrm{m}}\left(\mathrm{J} \mathrm{mol}^{-1} \mathrm{~K}^{-1}\right)$ & 7.63 & 9.16 & 10.12 \\
$\kappa\left(10^{-12} \mathrm{~Pa}^{-1}\right)[21]$ & 5.889 & 5.510 & 5.640 \\
$f\left(\mathrm{~J} / \mathrm{m}^{2}\right)$ & 3.69 & 4.56 & 3.984 \\
$\gamma\left(\mathrm{J} / \mathrm{m}^{2}\right)[22]$ & 2.43 & 2.78 & 2.01 \\
$\gamma_{\mathrm{ss}}\left(\mathrm{J} / \mathrm{m}^{2}\right)$ & 0.275 & 0.407 & 0.491 \\
$\gamma^{\prime}\left(\mathrm{J} / \mathrm{m}^{2}\right)$ & 0.249 & 0.364 & 0.406 \\
$C$ & 12.80 & 13.52 & 15.39 \\
\hline
\end{tabular}

The relevant parameters in Figures 1 and 2 are listed in Table 1, where the magnetic constant $\mu_{0}=4 \pi \times 10^{-7} \mathrm{H} / \mathrm{m}[16]$; For metals, $S_{\mathrm{vib}} \approx S_{\mathrm{m}}=H_{\mathrm{m}} / T_{\mathrm{m}}$ [17]; $\kappa=1 / B$, with $B$ being the bulk modulus [12]; The $f$ and $\gamma_{\mathrm{ss}}$ were computed by formulas (7) and (8) with $T=298 \mathrm{~K}$; the value of $\gamma^{\prime}$ was calculated with the $\gamma_{\mathrm{ss}}$ values of the substrate being $0.222 \mathrm{~J} / \mathrm{m}^{2}$ and $0.320 \mathrm{~J} / \mathrm{m}^{2}$ for $\mathrm{Ag}$ and $\mathrm{Cu}$, respectively [12].

\section{CONCLUSIONS}

Considering the critical size and material shape, this paper puts forward a size-dependence model of the Curie temperature $T_{\mathrm{c}}$ of uniform low-dimensional ferromagnetic materials, based on energy theory and thermodynamics. The proposed model, with no adjustable free parameter, was used to predict the effect of material size on the Curie temperature, revealing that the $T_{\mathrm{c}}$ value decreases with the material size. This result agrees well with the experimental results and the prediction by other theoretical models.

\section{ACKNOWLEDGEMENT}

This paper is made possible thanks to the support from the National Natural Science Foundation of China (Grant No.: 51878316).

\section{REFERENCES}

[1] Shen J, Kirschner J. (2002). Tailoring magnetism in artificially structured materials: The new frontier. Surface Science 500(1-3): 300-322. https://doi.org/10.1016/S0039-6028(01)01557-6

[2] Zhang R, Willis RF. (2001). Thickness-dependent Curie temperature of ultrathin magnetic films: effect of the range of spin-spin interactions. Physical Review Letters 86(12): https://doi.org/10.1103/PhysRevLett.86.2665 $2665-2668$

[3] Zhong WH, Sun CQ, Tay BK, Bai HL, Jiang EY. (2002). Curie temperature suppression of ferromagnetic nanosolids. Journal of Physics: Condensed Matter 14(23): L399-L405. https://doi.org/10.1088/0953$8984 / 14 / 23 / 101$

[4] Du YW, Xu MX, Wu J, Shi YB, Lu HX, Xue RH. (1991). Magnetic properties of ultrafine nickel particles. Journal of Applied Physics 70: 5903-5905. https://doi.org/10.1063/1.350101

[5] Huang H, Sun CQ, Hing P. (2000). Surface bond contraction and its effect on the nanometeric sized lead zirconate titanate. Journal of Physics: Condensed Matter 12(6): L127-L132. https://doi.org/10.1088/0953$8984 / 12 / 6 / 111$

[6] Jiang Q, Shi HX, Li JC. (1999). Finite size effect on glass transition temperature. Thin Solid Films 354(1-2): 283286. https://doi.org/10.1016/S0040-6090(99)00537-4

[7] Zhang Z, Zhao M, Jiang Q. (2001). Glass transition thermodynamics of organic nanoparticles. Physica B 293(3-4): 232-236. https://doi.org/10.1016/s09214526(00)00564-0

[8] Kittel C. (1949). Physical theory of ferromagnetic domains. Review of Modern Physics 21(4): 541-583. https://doi.org/541.10.1103/RevModPhys.21.541

[9] Needs RJ, Godfrey MJ. (1990). Surface stress of aluminum and jellium. Physical Review B 42(17): 10933-10939.

https://doi.org/10.1103/PhysRevB.42.10933

[10] Jiang Q, Liang LH, Zhao DS. (2001). Lattice contraction and surface stress of fcc nanocrystals. The Journal of Physical Chemistry B 105(27): 6275-6277. https://doi.org/10.1021/jp010995n 
[11] Suzuki T, Harada K, Honda N, Ouchi K. (1999). Preparation of ordered Fe-Pt thin films for perpendicular magnetic recording media. Journal of Magnetism and Magnetic Materials 193(1-3): 85-88. https://doi.org/10.1016/S0304-8853(98)00407-7

[12] Jiang Q, Liang LH, Zhao DS. (2001). Lattice contraction and surface stress of fcc nanocrystals. The Journal of Physical Chemistry B 105(27): 6275-6277. https://doi.org/ 10.1021/jp010995n

[13] Jiang Q, Zhao D S, Zhao M. (2001). Size-dependent interface energy and related interface stress. Acta materialia $\quad 49(16)$ : 3143-3147. https://doi.org/10.1016/S1359-6454(01)00232-4

[14] Sun L, Searson PC, Chien CL. (2000). Finite-size effects in nickel nanowire arrays. Physical Review B 61(10): R6463-R6466. https://doi.org/10.1103/PhysRevB.61.R6463

[15] Valiev RZ, Vishnyakov YD, Mulyukov RR, Fainshtein GS. (1990). On the decrease of Curie temperature in Submicron-Grained nickel. Physica Status Solid 117(2): 549-553. https://doi.org/10.1002/pssa.2211170226

[16] Mansfield M, Needs RJ. (1991). Surface energy and stress of lead (111) and (110) surfaces. Physical Review B 43(11): 8829-8833. https://doi.org/10.1103/PhysRevB.43.8829

[17] Jiang Q, Wang YW, Li JC. (1999). Thermodynamics of formation of carbon nanotubes catalyzed by nickel. Appl. Surface $\quad$ Science 152(3-4): $156-160$ https://doi.org/10.1016/S0169-4332(99)00327-X

[18] Buff FP. (1951). The spherical interface. I. Thermodynamics. The Journal of Chemical Physics 19(12): 1591-1594. https://doi.org/10.1063/1.1748127

[19] Langmuir I. (1916). The constitution and fundamental properties of solids and liquids. Journal of the American Chemical Society 38(11): 2221-2295. https://doi.org/10.1016/s0016-0032(17)90938-x

[20] Streitz FH, Cammarata RC, Sieradzki K. (1994). Surface-stress effects on elastic properties. II. Metallic multilayers. Physical Review B 49(15): 10707-10716. http://link.aps.org/doi/10.1103/PhysRevB.49.10707

[21] Hoyt JJ, Asta M, Karma A. (2003). Atomistic and continuum modeling of dendritic solidification. Materials Science and Engineering: R: Reports 41(6): 121-163. https://doi.org/10.1016/j.cossms.2015.09.001

[22] Vitos L, Ruban AV, Skriver HL, Kollar J. (1998). The surface energy of metals. Surface Science 411(1-2): 186202. https://doi.org/10.1016/S0039-6028(98)00363-X

[23] Qiu ZQ, Person J, Bader SD. (1993). Asymmetry of the spin reorientation transition in ultrathin $\mathrm{Fe}$ films and wedges grown on Ag (100). Physical Review Letters 70(7): 1006-1009. https://doi.org/10.1103/PhysRevLett.70.1006

[24] Huang F, Kief MT, Mankey GJ, Willis RF. (1994). Magnetism in the few-monolayers limit: A surface magneto-optic Kerr-effect study of the magnetic behavior of ultrathin films of $\mathrm{Co}, \mathrm{Ni}$, and $\mathrm{Co}-\mathrm{Ni}$ alloys on $\mathrm{Cu}$ (110) and $\mathrm{Cu}$ (111). Physical Review B 49(6): 3962-3971. https://doi.org/10.1103/PhysRevB.49.3962

[25] Schneider CM, Bressler P, Schuster P, Kirschner J. (1990). Curie temperature of ultrathin films of fcc-cobalt epitaxially grown on atomically flat $\mathrm{Cu}(100)$ surfaces. Physical Review Letters 64(9): 1059-1062. https://doi.org/10.1103/PhysRevLett.64.1059

[26] Huang F, Mankey GJ, Kief MT, Willis RF. (1993). Finite-size scaling behavior of ferromagnetic thin films. Journal of Applied Physics 73(10): 6760-6762. https://doi.org/ 10.1063/1.352477

[27] Tischer M, Arvanitis D, Yokoyanma T, Lederer T, Troger L, Baberschke K. (1994). Temperature dependent MCXD measurements of thin Ni films on $\mathrm{Cu}$ (100). Surface Science 307-309: 1096-1101. https://doi.org/10.1016/0039-6028(94)91546-6

[28] Roelofs A, Schneller T, Szot K, Waser R. (2003). Towards the limit of ferroelectric nanosized grains. Nanotechnology 14(2): https://doi.org/10.1088/0957-4484/14/2/328 